\title{
ЕСТЬ ЛИ МЕСТО ЛУЧЕВОЙ ТЕРАПИИ В ЛЕЧЕНИИ МЕСТНОРАСПРОСТРАНЕННОГО РАКА ЯИЧНИКОВ
}

\section{IS THERE A PLACE FOR RADIATION THERAPY IN THE TREATMENT OF LOCALLY ADVANCED OVARIAN CANCER}

\author{
T. Sharabura \\ V. Moiseyenko \\ A. Khadzhimba \\ D. Scherbatykh
}

Summary. The generally accepted standard treatment for epithelial ovarian cancer is currently performing optimal cytoreductive surgery with adjuvant chemotherapy with platinum and taxanes. Primary treatment ensures complete clinical remission in more than $70 \%$, but most patients with a locally advanced process experience a relapse of the disease, mainly with intra-abdominal localization. Adjuvant radiation therapy with pelvic abdominal radiation used in the 1950s was inferior to platinum-containing chemotherapy in efficacy and toxicity and was excluded from recommendations for the treatment of ovarian cancer. Modern technologies of radiation therapy make it possible to take a fresh look at the capabilities of the method both for consolidating remission after first-line therapy and for treating relapses of the disease.

Keywords: ovarian cancer, radiation therapy, treatment.
Шарабура Татьяна Михайловна

К.м.н., ГБУЗ «Санкт-Петербургский кинический научно-практический чентр специализированных видов медицинской помощи(онкологический)» tatyana1612@yandex.ru

Моисеенко Владимир Михайлович Д.м.н., профессор, директор, СанктПетербургский кинический научно-практический центр специализированных видов медицинской помощи(онкологический)» moiseyenkov@gmail.com

Хаджимба Анжелла Славиковна

Д.м.н., Санкт-Петербургский кинический научнопрактический чентр специализированных видов медичинской помощи(онкологчческий)» khadzhimba@gmail.com

Щербатых Дарья Евгеньевна Н.с., Санкт-Петербургский научно-клинический центр специализированных видов медицинской помоши (онкология) scherbatykh.d@bk.ru

Аннотация. Общепризнанным стандартном лечения эпителиального рака яичников в настоящее время является выполнение оптимальной циторедуктивной операции с адъювантной химиотерапией препаратами платины и таксанами. Первичное лечение обеспечивает достижение полной клинической ремиссией более, чем в 70\%, но у большинства пациенток с местнораспространенным процессом возникает рецидив заболевания, преимущественно с интраабдоминальной локализацией. Применявшаяся в 1950-е годы адъювантная лучевая терапия с тазово-абдоминальным облучением уступала платиносодержащей химиотерапии по эффективности и токсичности и была исключена из рекомендаций по лечению рака яичников. Современные технологии лучевой терапии позволяют по-новому взглянуть на возможности метода как для консолидации ремиссии после терапии первой линии, так и для лечения рецидивов заболевания.

Ключевые слова: рак яичников, лучевая терапия, лечение.

При комплексном лечении распространенного РЯ, включающем операцию и химиотерапию на основе препаратов платины и таксанов, у значительной части больных удается достичь полного ответа. К сожалению, ремиссии редко бывают длительными, а эффективность последующих схем химиотерапии снижается. Для улучшения выживаемости необходим поиск новых стратегии лечения. Особенностью течения РЯ является преобладание трансперитонеальной диссеминации, у 85\% пациенток распространение опухоли 
ограничивается брюшной полостью и полостью малого таза. Послеоперационная лучевая терапия исторически использовалась для лечения рака яичников всех подтипов с хорошими результатами $[2,3]$. Несмотря на радиочувствительность рака яичников, применение лучевой терапии в лечении данной патологии в настоящее время ограничено.

Применение лучевой терапии (ЛТ) в лечении РЯ имеет долгую и непростую историю. В 1950-е годы, до эры химиотерапии, операция с адъювантной лучевой терапией была основным методом лечением данной патологии. В связи с преимущественным распространением эпителиального рака яичников в пределах брюшной полости и малого таза, преобладанием интраабдоминальных рецидивов облучению подлежала вся брюшная полость и малый таз. Попытки ограничиться облучением только малого таза, даже при ранних стадиях, оказались неэффективными [4].

\section{ААъювантное тазово-абАоминальное облучение}

B 1992 г. Dembo A.J. проанализировал накопленный опыт ЛТ с тазово-абдоминальным облучением (ТАО) рака яичников I - IV стадий: применявшиеся в то время методики облучения брюшной полости «смещающимися полосами» и открытыми полями показали сопоставимую эффективность и токсичность, но предпочтение было отдано облучению открытыми полями в связи с более упрощенной техникой. Независимо от техники облучения наиболее тяжелыми и частыми были осложнения, связанные с кишечной непроходимостью, потребовавшие в 5,6\% (от 1,4\% до 14\%) хирургического лечения. Риск кишечной непроходимости был выше при дозах на малый таз более 45 Гр и дозах на всю брюшную полость (БП) более 25 Гр. Наилучший результат от адъювантной ЛТ с ТАО был получен у пациенток промежуточного риска (II-III ст., Grade1 и I-II ст., Grade 2-3) и при отсутствии макроскопической опухоли в верхнем отделе брюшной полости и небольшой (менее 2 см) макроскопической опухолью в малом тазу. 5-летняя общая выживаемость (ОВ) в этой группе составила 75\%. У пациенток высокого риска (II Ст., Grade 3 и III ст., Grade 2-3) адъювантная ЛТ с ТАО оказалась менее эффективной, 10-летняя выживаемость без рецидива (ВБР) составила лишь 20\% [4].

Осложнения ЛТ с облучением всей брюшной полости и неудовлетворительные результаты у пациенток c III стадией послужили основанием для поиска альтернативных методов лечения. Последующий прогресс в лечении РЯ связан с химиотерапией (ХТ). В 1970-е годы XT рака яичников основывалась на применении алкилирующих препаратов (мелфалан, хлорамбуцил, тиофосфамид), результаты применения которых оказа- лись сопоставимы с адъювантной ЛТ с ТАО при меньшей токсичности XT [5]. С появлением препаратов платины началась новая эра в лечении РЯ. Платиносодержащая XT оказалась более эффективной, менее токсичной и более доступной в сравнении с ЛТ при адъювантном лечении РЯ I-III стадий [6, 7]. С этого времени ЛТ с ТАО стала все меньше использоваться, а с 2007 г. была исключена из рекомендаций по лечению рака яичников NCCN (National Comprehensive Cancer Network). Стандартным вариантом лечения РЯ в настоящее время является выполнение оптимальной циторедуктивной операции с адъювантной химиотерапией препаратами платины и таксанами [6].

Вместе с тем, проблема лечения пациенток с местно-распространенным процессом и редкими несерозными гистологическими типами рака яичников по-прежнему остается актуальной. В 2016 г был опубликован анализ базы данных SEER (Surveillance, Epidemiology, and End Results) с 2004 по 2011 годы, включавший 15829 пациенток со светлоклеточной, муцинозной и эндометриоидной карциномами яичников I-III стадиями, из которых 450 была проведена адъювантная ЛТ. Отмечено улучшение 10 -летней ОВ у пациенток с III стадией, получивших адъювантную ЛТ (36\% против 30\%), а наилучший результат был у пациенток с муцинозным гистотипом, 10-летняя ОВ составила $45 \%$ с ЛТ и 26\% - без облучения [7]. Эти результаты свидетельствуют о том, что роль ЛТ при местно-распространенном РЯ требует дальнейшего изучения, хотя возврат к адъювантному тазово-абдомнальному облучению маловероятен.

\section{Консолихируюшая терапия}

«Золотым стандартом» лечения РЯ является ХТ таксанами и платиной, что, впрочем, не решило проблему высокого риска рецидивов. Более, чем у 70\% пациенток с III стадией РЯ в течение двух лет после терапии первой линии развиваются рецидивы [8]. В этой связи следующей задачей стал поиск методов консолидирующей или поддерживающей терапии, продляющих ремиссию после операции и адъювантной ХТ. Несмотря на токсичность, были предприняты попытки использовать тазово-абдоминальное облучение с консолидирующей целью. В австрийском рандомизированном исследовании (1999 г.) консолидирующая ЛТ с ТАО при полном клиническом ответе после 6 циклов адъювантной ХТ улучшила ВБР и ОВ в сравнении с наблюдением. Наибольший выигрыш был у пациенток с III стадией, 5-летняя ВБР выросла с 19\% до 45\% (p = 0.0061), а 5-летняя ОВ - с 26\% до $59 \%(p=0.012)$ [9].

Может ли консолидирующая ЛТ с ТАО конкурировать с поддерживающей ХТ. Этот вопрос был изучен 
в нескольких рандомизированных исследованиях. В исследовании Swedish-Norwegian OCSG 172 пациентки с III стадией РЯ после оптимальной циторедукции и адъювантной ХT были рандомизированы на три группы: получивших ТАО с дозой 20 Гр за 20 фракций и буст на малый таз до 40,4 Гр; получивших XТ (6 циклов

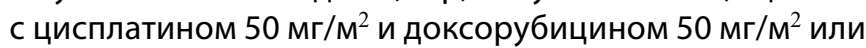

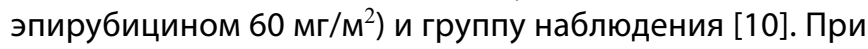
полном морфологическом ответе, подтвержденном морфологически («second look surgery»), 5-летняя выживаемость без прогрессии (ВБП) составила 56\%, 36\% и $35 \%$, соответственно ( $p=0,032), 5$-летняя ОВ в группе с ЛТ также оказалась выше и составила 69\% в сравнении с 57\% в группе с XТ, но при наличии остаточной микроскопической опухоли не было достоверных различий между ЛТ и ХТ. В группе с ЛТ было меньше рецидивов, но выше токсичность, поздние кишечные осложнения Grade 3 составили 10\%. Автор исследования считает, что ТАО с консолидирующей целью при полном морфологическом ответе после ХТ имеет перспективы при условии снижения токсичности [10].

К сожалению, другим авторам не удалось подтвердить эти результаты $[11,12.13]$. В рандомизированном исследовании North Thames Ovary Group сравнили эффективность консолидирующей терапии с ТАО (24 Гр) и ХТ с карбоплатином (5 циклов). В исследование было включено 117 пациенток с IIB-IVстадиями РЯ после операции и XT с карбоплатином с резидуальной опухолью $\leq 2$ см [11]. Авторы отметили хорошую переносимость и низкую токсичность в обеих группах, но был случай смерти, связанный осложнением после Лт. 5-летняя ОВ оказалась низкой в обеих группах и составила 32\%. Аналогичные результаты получены в исследовании West Midlands Trial II. Lawton F. et al. рандомизировали 109 пациенток с IIB-IV стадиями РЯ на получивших консолидирующую ЛТ (доза на БП составила 22,5 Гр и на малый таз - 45 Гр) или хлорамбуцил в течение года. 2-х летняя ОВ составила $35 \%$, токсичность оказалась высокой в обеих группах, лишь половина всех пациенток смогли завершить лечение. Авторы считают, что нет оснований для консолидирующей ЛТ или ХТ хлорамбуцилом после операции и XТ с цисплатином [12]. Еще одно рандомизированное исследование (GONA) было остановлено досрочно из-за явных преимуществ консолидирующей XT над TAO. 41 пациентка с III-IV стадиями РЯ после операции и ХТ с полным клиническим ответом были рандомизированы на получивших ТАО (доза на БП составила 30,2 Гр и 43,2 Гр - на малый таз) или 3 цикла ХТ на основе препаратов платины. При медиане наблюдения 1,8 года прогрессирование отмечено у 55\% больных в группе с ЛТ и у 29\% в группе с ХТ [13].

Мета-анализ 2010г, включавший 29 исследований, показал, что консолидирующая и поддерживающая химиотерапия улучшают ОВ и ВБП в сравнении с наблюдением, а наилучший результат следует ожидать у пациенток с распространенными стадиями и полным клиническим или патоморфологическим ответом после терапии первой линии [14]. Однако, в Кокрейновском обзоре 2013г, включавшем 8 рандомизированных исследований и 1644 пациентки РЯ, не удалось получить убедительных данных, подтверждающих влияние поддерживающей XT препаратами платины, доксорубицином или паклитакселом на общую выживаемость в сравнении с наблюдением [15].

Следует признать, что на сегодняшний день проблема продления безрецидивного периода после терапии первой линии при распространенном РЯ не решена. Последнее объясняет сохраняющийся интерес к ЛТ с ТАО и попытки справиться с токсичностью за счет поиска оптимальной дозы [16]. В 1998 г. Fyles A.W. et al. опубликовали результаты рандомизированного исследования, показавшего, что ТАО с дозой 22,5 Гр и 27,5 Гр сопоставимо по показателям 5-летней ОВ и ВБП. Однако этого уровня доз оказалось недостаточно для обеспечения высокого локального контроля, 72\% всех рецидивов локализовалось в малом тазу и/или брюшной полости [17]. Полученные результаты послужили основанием для последующих исследований с эскалацией дозы. Firat S. et al. в 2003г опубликовали отдаленные результаты высокодозного облучения брюшной полости у 71 пациентки с I-III стадиями РЯ после операции с или без ХТ. Средняя доза на БП составила 36 Гр (9-45,5 Гр), на малый таз - 51 Гр (16-59 Гр). Увеличение дозы на брюшную полость $\geq 36$ Гр снизило риск абдоминальных рецидивов с 49\% до $18 \%$ ( $\mathrm{p}=0.006)$, но увеличило риск кишечной непроходимости. В $11 \%$ случаев потребовалось хирургическое лечение тонкокишечной непроходимости. Авторы исследования уверены, что перспективы ЛТ рака яичников связаны с возможностью снижения риска осложнений. [18].

\section{Аучевая терапия

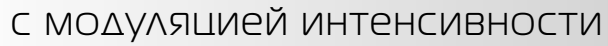 \\ и стереотаксическая $\wedge \top$}

Технические возможности контроля токсичности ЛТ появились уже в новом веке. Высокопрецизионные технологии, в частности лучевая терапия с модуляцией интенсивности (ЛТМИ) и стереотаксическая ЛТ (СТЛТ), обеспечили точное подведение дозы при хорошей защите органов риска. В 2010 году Rochet N. et al. onyбликовали результаты исследования I фазы, в котором 10 пациенток C III стадией рака яичников после оптимальной циторедуктивной операции и адъювантной XТ с карбоплатином и таксанами получили с консолидирующей целью ЛТМИ с подведением на всю брюшную полость 30 Гр за 20 фракций по 1,5 Гр. лтМИ обеспечила 
хорошее распределение дозы в пределах всей брюшной полости, включая области наиболее частых рецидивов, такие как капсула печени, диафрагма, парааортальные и тазовые лимфатические узлы. В сравнении c 3-D конформной ЛТ удалось снизить дозу на печень, почки и костный мозг. При медиане наблюдения 23 месяца диагностированы три случая интраперитонеальных рецидивов и один случай метастазов в печень.

Однако поздних осложнений избежать не удалось. Тонкокишечная непроходимость развилась у трех пациенток через 2,5, 3 и 13 месяцев после лт [19]. Тем не менее, авторы посчитали опыт успешным и инициировали в 2011 году исследование II фазы OVAR-IMRT-02, В которое были включены 37 пациенток с III стадией PЯ после оптимальной циторедуктивной операции и адъювантной XT, получившие полный ответ после лечения. Консолидирующая ЛТМИ проводится по аналогичной схеме с подведением на всю брюшную полость 30Гр за 20 фракций по 1,5Гр в течение 4 недель [20]. Rochet N. et al. отказались от дополнительного облучения малого таза в связи с риском увеличения кишечной токсичности и отсутствием убедительных данных улучшения выживаемости. До настоящего времени окончательные результаты этого исследования не опубликованы.

Вместе с тем, очевидно, что ТАО, в том числе с применением современных технологий, неизбежно требует ограничения дозы, что не позволяет в полной мере реализовать возможности данного метода. Последнее подтверждается результатами паллиативной терапии с локальным подведением тумороцидных или близких к ним доз. В исследовании Machida S. et al. у 33 пациенток с локо-регионарными рецидивами рака яичников удалось облучить все опухолевые очаги одним полем с использованием 3-D конформной лТ с медианой дозы 60 Гр (42-65 Гр). Получен ответ в 66\% случаев с локальным контролем - 100\%. Медиана выживаемости до прогрессии составила 10 месяцев, а медиана ОВ - 20 месяцев. Облучение локализованных рецидивов с дозой 50-60 Гр оказалось эффективнее предшествующей XT [21]. Brown A.P. et al. показали возможности облучения вовлеченными полями (involved field RT) рецидивов в лимфатических узлах и экстранодальных рецидивов рака яичников с дозой 45Гр. 5-летний локальный контроль (ЛК) составил 71\% [22].

Применение ЛТ при рецидивах после предшествующей ХТ было ограничено из-за представлений о наличии перекрестной резистентности к ЛТ и препаратам платины. Choi N. et al. изучили эффективность Лт рецидивов РЯ со средней биологической эффективной дозой (БЭД) 50,7 Гр (от 28,0 до 79,2 Гр). 1-летний и 2-летний локальный контроль составили 66\% и 55\%, соответственно. ЛК был выше при облучении локо-ре- гиональных рецидивов в сравнении с отдаленными метастазами и при БЭД 50 Гр и более, при этом оказалось, что резистентность к ХТ, хотя и увеличивает риск прогрессии после ЛТ, но не коррелирует с возможным ответом на облучение.

Новые возможности безопасной эскалации дозы при облучении локализованных рецидивов или метастазов связаны с СТЛТ, что позволило добиваться длительной ремиссии у пациентов с олигометастазами злокачественных новообразований гинекологического профиля, включая рак яичников [23, 24]. Mesko S. et al. оценили результаты СТЛТ с медианой дозы 40Гр за 5 фракций. Положительный ответ был получен в 83\% случаев с достижением полного ответа в $17 \%$. Эффективность была выше при опухолях менее 2 см и большем значении БЭД (79,0 против 59,6 Гр, $p=0.027)$ [23]. Laliscia C. et al. получили полный ответ в $64 \%$ случаев после СТЛТ с дозой 24Гр за 1 фракцию или 27Гр за 3 фракции. Все пациентки с рецидивами РЯ получили ХТ после СТЛТ [24]. Преимущества СТЛТ наиболее полно реализуются при метастазах в регионарные лимфатические узлы, возможности конвенционального облучения которых ограничены из-за близости органов риска [25]. Достижение высокого локального контроля не исключает прогрессию заболевания вне поля облучения, что определило необходимость сочетания локального облучения рецидивов с XT. Kunos C.A. et al. провели исследование I фазы для оценки безопасности XT до СТЛТ с тазово-абдоминальными рецидивами, в том числе у пациенток с рецидивами РЯ. В первый день лечения вводился карбоплаптин (AUC2 или 4) и гемцитабин (600-800 мг/м $\left.{ }^{2}\right)$ и с 2 по 4 дни проводилась СТЛТ с дозой 24Гр за 3 фракции по 8 Гр. Частичный ответ получен в 79\%, стабилизация - в 21\% [26]. Полученные результаты свидетельствуют о эффективности и низкой токсичности высокопрецизионного и высокодозного облучения рецидивов, обеспечивающего не только стойкий локальный контроль, но и, возможно, оказывающего системное действие за счет абскопального эффекта [25].

\section{Зак^ючение}

Местно-распространенный рак яичников остается труднонеизлечимым заболеванием с частыми рецидивами. Следует признать, что несмотря на успешную терапию первой линии в большинстве случаев, не решена проблема обеспечения стойкого локо-регионарного контроля. Усовершенствованные лучевые методы с более низкой токсичностью привели к возобновлению интереса к использованию лучевой терапии для лечения метастатического рака в различных локализациях, включая рак яичников. Токсичность ограничивала использование этого метода лечения. Однако 
понимание молекулярных различий гистологических подтипов рака яичников с дифференцированном ответом как на химиотерапию, так и на лучевое лечение возобновило новый интерес к потенциальному применению лучевой терапии. Очевидно, также, что облучение всей брюшной полости не может конкурировать с платиносодержащей ХТ в адъювантном режиме, но, с учетом возможностей современных технологий лучевой терапии, роль ТАО при местно-распространенном РЯ требует дальнейшего изучения. Локальное облучение рецидивов РЯ имеет все основания для более широкого и раннего применения с целью продления жизни больных. При возможности высокодозного облучения всех интраабдоминальных рецидивов вероятность достижения полной и длительной ремиссии возрастет.

\section{ЛИТЕРАТУРА}

1. Состояние онкологической помощи населению России в 2017 г. Под ред. А.Д. Каприна, В.В. Старинского, Г.В. Петровой // М.: МНИОИ им. П.А. Герцена филиал ФГБУ «НМИЦ радиологии» Минздрава России, 2018. - илл. - 236 с

2. National Comprehensive Cancer Network. NCCN clinical practice guidelines in oncology: ovarian cancer including fallopian tube cancer and primary peritoneal cancer (version 2.2018) [Internet].

3. Redman C.W., Mould J., Warwick J. et al. The West Midlands epithelial ovarian cancer adjuvant therapy trial. //Clin Oncol (R Coll Radiol). 1993;5(1):1-5. doi:10.1016/s0936-6555(05)80682-x

4. Dembo A.J. Epithelial ovarian cancer: the role of radiotherapy. //Int J Radiat Oncol Biol Phys 1992, 22:835-845. doi:10.1016/0360-3016(92)90777-f.

5. Klaassen D, Shelley W, Starreveld A, Kirk M, Boyes D, Gerulath A, et al. Early stage ovarian cancer: a randomized clinical trial comparing whole abdominal radiotherapy, melphalan, and intraperitoneal chromic phosphate: a National Cancer Institute of Canada Clinical Trials Group report. J Clin Oncol (1988) 6(8):1254-63. doi:10.1200/JC0.1988.6.8.1254

6. Chiara S., Conte P., Franzone P. High-risk early-stage ovarian cancer. Randomized clinical trial comparing cisplatin plus cyclophosphamide versus whole abdominal radiotherapy. // Am J Clin Oncol. 1994 Feb;17(1):72-6.doi:10.1097/00000421-199402000-00016

7. Patel S.C., Frandsen J., Bhatia S., Gaffney D. Impact on survival with adjuvant radiotherapy for clear cell, mucinous, and endometriod ovarian cancer: the SEER experience from 2004 to 2011. J Gynecol Oncol (2016) 27(5): e45. doi:10.3802/jgo.2016.27.

8. Ozols R.F., Bundy B.N., Greer B.E. et al. Phase III trial of carboplatin and paclitaxel compared with cisplatin and paclitaxel in patients with optimally resected stage III ovarian cancer: a Gynecologic Oncology Group study. //J Clin Oncol 2003, 21:3194-3200. doi:0.1200/JC0.2003.02.153

9. Pickel H., Lahousen M., Petru E. et al. Consolidation radiotherapy after carboplatin-based chemotherapy in radically operated advanced ovarian cancer. // Gynecol Oncol (1999) 72(2):215-9. doi:10.1006/gyno.1998.5184

10. Sorbe B. Consolidation treatment of advanced (FIGO stage III) ovarian carcinoma in complete surgical remission after induction chemotherapy: a randomized, controlled, clinical trial comparing whole abdominal radiotherapy, chemotherapy, and no further treatment. //Int J Gynecol Cancer. 2003 May-Jun;13(3):278-86. doi:10.1046/j.1525-1438.2003.13193.x

11. Lambert H.E., Rustin G.J., Gregory W.M., Nelstrop A.E. A randomized trial comparing single-agent carboplatin with carboplatin followed by radiotherapy for advanced ovarian cancer: a North Thames Ovary Group study. //J Clin Oncol. 1993 Mar;11(3):440-8.doi:10.1200/JC0.1993.11.3.440

12. Lawton F., Luesley D., Blackledge G. et al. A randomized trial comparing whole abdominal radiotherapy with chemotherapy following cisplatinum cytoreduction in epithelial ovarian cancer. West Midlands Ovarian Cancer Group Trial II.//Clin Oncol (R Coll Radiol). 1990 Jan;2(1):4-9. doi:10.1016/s0936-6555(05)80210-9

13. Bruzzone M., Repetto L., Chiara S. et al. Chemotherapy versus radiotherapy in the management of ovarian cancer patients with pathological complete response or minimal residual disease at second look. //Gynecol Oncol. 1990 Sep;38(3):392-5.

14. Hess L.M., Rong N., Monahan P.O. et al. Continued Chemotherapy After Complete Response to Primary Therapy Among Women With Advanced Ovarian Cancer: A Meta-Analysis. // Cancer 2010 November 15; 116(22): 5251-5260. doi:10.1002/cncr.25487.

15. Mei L., Chen H., Wei DM. et al. Maintenance chemotherapy for ovarian cancer. //Cochrane Database Syst Rev. 2013 Jun 29;(6) doi: 10.1002/14651858.CD007414. pub3

16. Choi N., Chang J.H., Kim S., Kim H.J. Radiation for persistent or recurrent epithelial ovarian cancer: a need for reassessment. // Radiat Oncol J 2017;35(2):144152. doi: 10.3857/roj.2017.00213.

17. Fyles A.W., Thomas G.M., Pintilie M. et al. A randomized study of two doses of abdominopelvic radiation therapy for patients with optimally debulked Stage I ( $n=43)$, II ( $n=71)$, and III ( $n=11$ ) ovarian cancer. //Int J Radiat Oncol Biol Phys. 1998 Jun 1;41(3):543-9.doi:10.1016/s0360-3016(98)00066-2

18. Firat S., Murray K., Erickson B. High-dose whole abdominal and pelvic irradiation for treatment of ovarian carcinoma: long-term toxicity and outcomes. // Int J Radiat Oncol Biol Phys. 2003 Sep 1;57(1):201-7.doi: 10.1016/s0360-3016(03)00510-8.

19. Rochet N., Sterzing F., Jensen A.D., Dinkel J., Herfarth K.K., Schubert K., et al. Intensity-modulated whole abdominal radiotherapy after surgery and carboplatin/ taxane chemotherapy for advanced ovarian cancer: phase I study. //Int J Radiat Oncol Biol Phys 2010, 76:1382-1389.doi: 10.1016/j.ijrobp.2009.03.061

20. Rochet N., Kieser M., Sterzing F. et al. Phase II study evaluating consolidation whole abdominal intensity-modulated radiotherapy (IMRT) in patients with advanced ovarian cancer stage FIGO III — The OVAR-IMRT-02 Study. //BMC Cancer 2011, 11:41 doi: 10.1186/1471-2407-11-41.

21. Machida S., Takei Y., Yoshida C., Takahashi Y., Koyanagi T., Sato N., et al. Radiation therapy for chemotherapy-resistant recurrent epithelial ovarian cancer. Oncology (2014) 86(4):232-8. doi: 10.1159/000357269 
22. Brown A.P., Jhingran A., Klopp A.H. et al. Involved-field radiation therapy for locoregionally recurrent ovarian cancer. //Gynecol Oncol. 2013 Aug; 130(2):300-5. doi: 10.1016/j.ygyno.2013.04.469.

23. Mesko S., Sandler K., Cohen J., Konecny G., Steinberg M., Kamrava M. Clinical outcomes for stereotactic ablative radiotherapy in oligometastatic and oligoprogressive gynecological malignancies. Int J Gynecol Cancer (2017) 27(2):403-8. doi: 10.1097/IGC.0000000000000869

24. Laliscia C. et al. Clinical Outcomes of Stereotactic Body Radiotherapy in Oligometastatic Gynecological Cancer. // Int J Gynecol Cancer. 2017 Feb;27(2):396-402. doi: 10.1097/IGC.0000000000000885

25. Fields E.C., McGuire W.P., Lin L., Temkin S.M. Radiation Treatment in women with Ovarian Cancer: Past, Present, and Future. //Front. 0ncol. 2017 7:177. doi: $10.3389 /$ fonc. 2017.00177 .

26. Kunos C.A., Sherertz T.M., MisImani M. et al. Phase I trial of carboplatin and gemcitabine chemotherapy and stereotactic ablative radiosurgery for the palliative treatment of persistent or recurrent gynecologic cancer. Front Oncol (2015) 5:126. doi: 10.3389/fonc.2015.00126.

( ) Шарабура Татьяна Михайловна ( tatyana1612@yandex.ru ), Моисеенко Владимир Михайлович ( moiseyenkov@gmail.com ),

Хаджимба Анжелла Славиковна ( khadzhimba@gmail.com ), Щербатых Дарья Евгеньевна ( scherbatykh.d@bk.ru ).

Журнал «Современная наука: актуальные проблемы теории и практики»

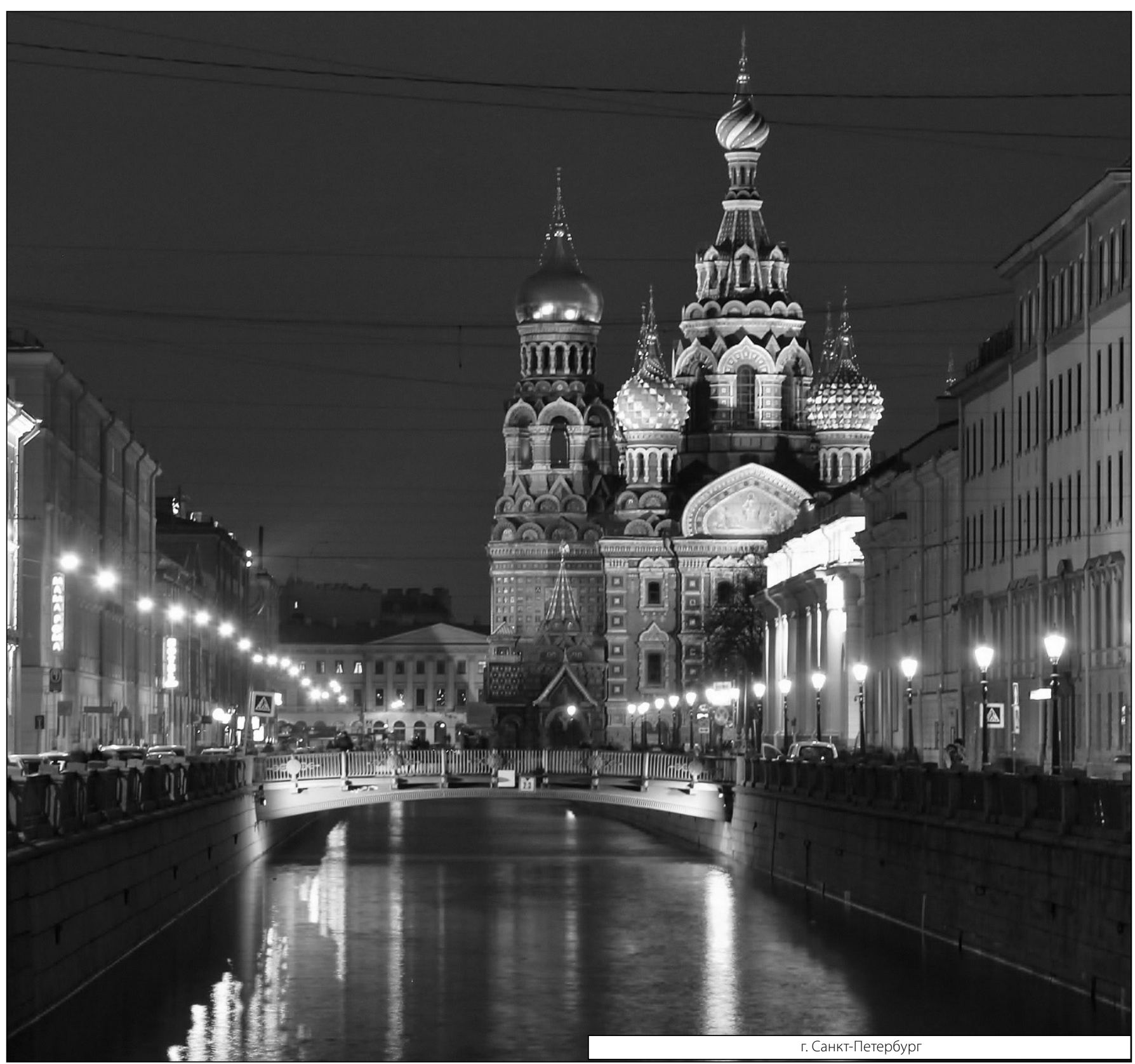

